\title{
COMPETÊNCIAS NA GESTÃO DE DESIGN DA INDÚSTRIA DO VESTUÁRIO
}

\author{
Cláudia de Souza Libânio \\ PPGEP/UFRGS, UniRitter, DEIS/UFCSPA \\ claudiasl@ufcspa.edu.br \\ Ana Paula Kloeckner \\ PPGEP/UFRGS \\ anapklt@yahoo.com.br \\ Laura Tasca \\ PPGEP/UFRGS \\ lauraltasca@gmail.com \\ Fernando Gonçalves Amaral \\ PPGEP/UFRGS \\ amaral@producao.ufrgs.br
}

Resumo: O Brasil está entre os maiores produtores de artigos têxteis do mundo. Considerando que o mercado de moda é altamente dinâmico, torna-se importante que haja uma resposta adequada a esse ambiente para atender às necessidades do mercado. Para isso, entender as competências e interações entre indivíduos e equipes torna-se determinante nesse processo. Desta maneira, este artigo tem por objetivo investigar o que pensam especialistas sobre competências em gestão de design, articulações e fatores intervenientes em duas empresas de grande porte inseridas na indústria do vestuário da região sul do Brasil. Por consequência, também busca conhecer como estão estruturadas as equipes de design e o relacionamento destas com a organização. A metodologia utilizada foi exploratória, qualitativa, por meio de entrevistas em profundidade com dois designers que trabalham nas referidas empresas. Posteriormente, foi feita uma análise das respostas, possibilitando a comparação dos dados obtidos com o referencial teórico pesquisado. Com este estudo, foi possível perceber que a principal causaraiz para a obtenção uma cultura de gestão de design nas empresas está relacionada às competências individuais e coletivas, conforme o caso estudado.

Palavras-chave: gestão de design; competências; interações; fatores intervenientes. 


\begin{abstract}
Brazil is among the largest producers of textiles in the world. Whereas the fashion market is highly dynamic, it is important that there is proper in this environment to meet the needs of the market response. For this, understand the competencies and interactions between individuals and teams becomes crucial in this process. Thus, this paper aims at investigate what experts think about competencies in design management, joints and intervening factors in two large companies inserted in the apparel industry of southern Brazil. Consequently, also seeks to know how design teams are structured and the relationship of these with the organization. The methodology was exploratory, qualitative, through indepth interviews with two designers who work in those organizations. Subsequently, an analysis of the responses was made, enabling the comparison of data obtained with the theoretical researched. With this study, it was revealed that the main cause for obtaining a culture of design management in enterprises is related to individual and collective competencies, as the case study.
\end{abstract}

Keywords: design management; competencies; interactions; intervenient factors.

\title{
1. INTRODUÇÃO
}

Inserida na cadeia produtiva têxtil e de confecção, a indústria do vestuário guarda características particulares, como: a proximidade com o consumidor final e o elevado número de agentes envolvidos em seus sistemas produtivos, por meio da terceirização. Este último fator, ao mesmo tempo em que exige alto controle gerencial do processo produtivo, possibilita uma celeridade no processo de desenvolvimento de produto.

No contexto mundial, o Brasil está entre os quatro principais países produtores de artigos têxteis (ABIT, 2010), e a Região Sul do país é a segunda maior produtora brasileira de confeccionados, ficando atrás somente da Região Sudeste (ABRAVEST, 2014). De acordo com o Ministério do Trabalho e Emprego, as mais de 98.000 indústrias formais no Brasil empregam mais de 600.000 trabalhadores (ABIT, 2010). Dentro deste contexto, o setor de vestuário do estado do Rio Grande do Sul representa mais de 7.500 destas indústrias e emprega em torno de 21.000 trabalhadores (ABIT, 2010). Para determinar a segmentação destes mercados, fatores como faixa etária, gênero, grupos sociais e nível de renda foram analisados (ABRAVEST, 2014).

Estudos internacionais evidenciam a importância da integração da equipe de design com os demais participantes de um projeto (REID et al., 2000; LAUCHE, 2005). Também aparecem conceitos de conhecimento compartilhado (KLEINSMANN; VALKENBURG, 2008), competências das organizações e dos profissionais (BRUCE et al., 1999; BELKADI et al., 2007), liderança nas equipes de design (LEE; CASSIDY, 2007) e do design como um agente de conhecimento e integração nas organizações (BERTOLA; TEIXEIRA, 2003; GIRARD; ROBIN, 2006) também aparecem como fatores relacionados a gestão de design. De acordo com Libânio e Amaral (2013), certos aspectos foram discutidos primeiramente no exterior, sendo debatidos no Brasil posteriormente. Ainda segundo os autores, características como liderança, autonomia, competências, empreendedorismo, pró-atividade, comunicação, integração e capacidade de trabalho 
em equipe foram elencadas como requisitos essenciais aos profissionais de design. Sendo assim, este estudo objetiva investigar o que pensam especialistas sobre competências em gestão de design, articulações e fatores intervenientes em duas empresas de grande porte inseridas na indústria do vestuário da região sul do Brasil. Por consequência, também busca conhecer como estão estruturadas as equipes de design e o relacionamento destas com a organização.

\subsection{Competências}

As competências individuais e coletivas estão ligadas aos arranjos internos e a estruturação e integração de equipes em organizações. O conceito de competência, de acordo com Dias et al. (2012, p. 12) "é focado predominantemente no desempenho, na mobilização contextualizada e na contribuição do trabalho para a estratégia da empresa." Retour (2012) define competência como um know-how operacional. Ruas (2005) afirma que a competência individual é fundamentada em um conjunto de capacidades, relacionando-se diretamente ao conhecimento, às habilidades e às atitudes do indivíduo envolvido no processo. O quadro 1 elenca as competências individuais com base nos conhecimentos, habilidades e atitudes.

Quadro 1 - Competências Individuais.

\begin{tabular}{|l|l|}
\hline $\begin{array}{l}\text { Competências } \\
\text { Individuais }\end{array}$ & Descrição \\
\hline Conhecimentos & técnicos, científicos, domínio de conceitos, cores \\
\hline Habilidades & $\begin{array}{l}\text { criatividade, pensamento estratégico, habilidades de apresentação, aptidões } \\
\text { comerciais }\end{array}$ \\
\hline Atitudes & $\begin{array}{l}\text { comprometimento, entusiasmo, autoconfiança, orientação para resultados, } \\
\text { construção de relacionamentos, resolução de problemas }\end{array}$ \\
\hline
\end{tabular}

Fonte: Adaptado de Ruas (2005) e Borja de Mozota (2003).

Já as competências coletivas, segundo Retour (2012), por seu caráter tácito e pela sua complexidade, são específicas das firmas e, por isso, dificilmente imitáveis. Retour e Krohmer (2006) elencam como atributos das competências coletivas o referencial comum, a linguagem compartilhada, a memória coletiva e o engajamento subjetivo, de acordo com o quadro 2. Já Michaux (2009) destaca que é mais apropriado levar em conta competências coletivas analisando, primeiramente, a natureza do grupo e a situação de trabalho.

Quadro 2-Competências Coletivas

\begin{tabular}{|l|l|}
\hline Competências Coletivas \\
\hline $\begin{array}{l}\text { Referencial } \\
\text { comum }\end{array}$ & $\begin{array}{l}\text { estrutura a ação coletiva que acaba por ser resultado da comparação entre } \\
\text { experiências e representações das pessoas envolvidas. }\end{array}$ \\
\hline $\begin{array}{l}\text { Linguagem } \\
\text { compartilhada }\end{array}$ & $\begin{array}{l}\text { vocabulário comum utilizado no trabalho que permite inclusive uma comunicação } \\
\text { cognitiva no momento da ação. }\end{array}$ \\
\hline Memória coletiva & aprendizagem (aprendizagens coletivas passadas e orienta as posteriores). \\
\hline $\begin{array}{l}\text { Engajamento } \\
\text { subjetivo }\end{array}$ & $\begin{array}{l}\text { mobilização subjetiva das pessoas orientadas para a empresa como um todo. } \\
\text { Resolução de imprevistos e algumas disfunções capazes de interromper o } \\
\text { andamento da ação. }\end{array}$ \\
\hline
\end{tabular}

Fonte: Adaptado de Retour e Krohmer (2006)

Ruas (2009) afirma que "competências coletivas da organização asseguram a realização da missão, da visão e/ou da estratégia da empresa". Borja de Mozota (2003) 
visualiza o design como um recurso e como uma competência central ou competênciachave. Retour (2012, p. 292) salienta que "as competências-chave são consideradas como essenciais e servem como apoio para se ter uma vantagem duradoura em termos de concorrência". Borja de Mozota (2003) ainda destaca que constituição de uma competência central na firma está pautada no desenvolvimento de novas capacidades de conhecimento, seja na esfera individual ou na organizacional.

\subsection{Estratégia aliada ao design}

Outro conceito que merece destaque é o conceito de estratégia. Mintzberg e Quinn (2001) evidenciam um caráter dinâmico, flexível e circunstancial ao processo de estratégia. Já Porter (1989) destaca que existem dois tipos de vantagem competitiva por custo e por diferenciação - que, relacionadas ao âmbito competitivo, determinam os distintos tipos de estratégias genéricas. Entretanto, o autor aponta que é relevante as empresas observarem, primordialmente, o ambiente externo para serem mais eficientes que os concorrentes (SILVA; LIBÂNIO; RUAS, 2013) mas não considera diferenças em uma mesma indústria nem aspectos internos específicos de cada organização.

Já Borja de Mozota (2003) relaciona estratégia ao design, defendendo que "a estratégia cria uma adequação entre as atividades da empresa. O sucesso da estratégia e do design estratégico depende de se fazerem muitas coisas bem e de integrá-las. 0 todo importa mais do que qualquer parte individual. "Strategic fit" é fundamental não somente para a vantagem competitiva, como também para a sustentabilidade dessa vantagem." (BORJA DE MOZOTA, 2003). Borja de Mozota (2003) acrescenta que é primordial a definição de uma estratégia empresarial que incorpore metas de design, a delimitação de uma estratégia de design e a garantia de que a estratégia de design reagrupe produtos, comunicação, ambiente e informações.

\section{METODOLOGIA}

A metodologia utilizada neste trabalho foi exploratória, segue a abordagem qualitativa, sendo conduzido por meio de pesquisa bibliográfica bem como de entrevistas em profundidade (MALHOTRA, 2012). Malhotra (2012) destaca que a pesquisa exploratória tem como principal foco auxiliar o pesquisador na compreensão da situação-problema. $\mathrm{O}$ autor ainda afirma, com relação à pesquisa qualitativa, que esta é uma metodologia de pesquisa não-estruturada, exploratória, a partir de pequenas amostras, possibilitando uma melhor percepção e compreensão do problema em questão. Yin (2001) salienta que o estudo de caso é utilizado como estratégia de pesquisa quando perguntas do tipo "como" e "por que" são colocadas, quando o pesquisador não controla os eventos e quando o foco da pesquisa está em fenômenos contemporâneos. A amostra é não probabilística, escolhida por conveniência.

Foi elaborado um roteiro semiestruturado para utilização nas entrevistas em profundidade. Dois designers que trabalham em grandes empresas pertencentes à indústria do vestuário foram entrevistados. Segundo Malhotra (2012), os especialistas são pessoas bem informadas a respeito do assunto e do setor de atuação. Os dados foram obtidos através de fontes primárias, sendo utilizado como procedimento de pesquisa uma abordagem direta e pessoal nas entrevistas. As entrevistas foram gravadas, transcritas e os dados tabulados para posterior análise. A partir dos dados 
coletados, foi realizada uma análise de conteúdo (BARDIN, 2005), buscando atingir os objetivos desta pesquisa. Para garantir o anonimato dos entrevistados, eles estão identificados como Especialista 1 (E1) e Especialista 2 (E2).

Para auxílio na análise das respostas, foi utilizado o software Mandala, que possibilitou verificar, através da análise das entrevistas, quais eram as palavras mais citadas e relacionadas pelos respondentes em relação ao tema em estudo. Os dados obtidos nessa etapa foram utilizados para a realização da Árvore da Realidade Atual (ARA).

A utilização dessa ferramenta do processo de pensamento da Teoria das Restrições (TOC) ocorreu para identificar claramente o problema central da situação em análise. A ARA é uma das cinco ferramentas do Processo de Pensamento (PP) da Teoria das Restrições proposta por Goldratt (1990). O PP pode ser considerado um método de identificação, análise e solução de problemas (LACERDA et al., 2009), no qual a ARA é a primeira ferramenta proposta, buscando identificar "o que mudar?". As cinco ferramentas (Quadro 3) podem ser utilizadas individualmente ou estarem ligadas logicamente, permitindo a identificação de problemas centrais, determinação de soluções tipo ganha-ganha e superação de obstáculos possíveis para implementação da solução. O objetivo da ARA é a definição dos problemas centrais encontrados em um sistema específico, proporcionando uma efetiva comunicação dos principais problemas da empresa e um entendimento comum desses problemas (LACERDA et al., 2009). Nela são apresentadas as relações de causa e efeito entre os efeitos indesejáveis, os quais são utilizados para diagnosticar o que no sistema precisa ser modificado (GUPTA et al., 2011).

Quadro 3 - Cinco ferramentas do processo de pensamento.

\begin{tabular}{|l|l|}
\hline Pergunta Central & Ferramenta \\
\hline O quê mudar? & Árvore da Realidade Atual \\
\hline Para o quê mudar? & $\begin{array}{l}\text { Evaporação das nuvens } \\
\text { Árvore da Realidade Futura }\end{array}$ \\
\hline Como provocar a mudança? & $\begin{array}{l}\text { Árvore de Pré-Requisitos } \\
\text { Árvore de Transição }\end{array}$ \\
\hline
\end{tabular}

Fonte: Adaptado de Lacerda et al. (2009)

\section{RESULTADOS E DISCUSSÃO}

A partir do objetivo proposto pelo artigo e dos dados coletados, foram desenvolvidas análises, no sentido de contribuir para uma discussão sobre o tema.

\subsection{Características das Empresas}

A empresa A é uma empresa de grande porte, terceiriza inteiramente a sua produção. Está há 49 anos no mercado e iniciou suas atividades como empresa familiar. Posteriormente, foi vendida a um grupo de investidores. Atende os públicos masculino, feminino e infantil, com mais de 200 lojas no Brasil. A empresa B é uma empresa familiar, de grande porte, atuando há 50 anos no mercado de roupas femininas. Tem produção própria, com uma fábrica nacional e outra internacional.

\subsection{Entrevistas em Profundidade - Caracterização da Gestão de Design nas Empresas}

A partir das informações obtidas através das entrevistas, buscou-se categorizar as informações obtidas como forma de comparar a situação das duas empresas em relação à gestão de design, competências e integração das equipes e pessoas. De uma 
maneira geral, foi possível observar que existe uma diferença na estruturação dessa área nas duas empresas (Quadro 5). O E1 ocupa o cargo de gerente de desenvolvimento técnico de produtos da empresa, sendo responsável pelo departamento de Estilo de Desenvolvimento de Produtos da companhia. A empresa A apresenta a área bem estruturada, assim como a disseminação dessa cultura através de seus colaboradores. Os processos de gestão de design são claros e a busca de integração e participação de pessoas todos os níveis hierárquicos e de um entendimento comum são explicitados durante a entrevista.

Quadro 5 - Caracterização da gestão de design nas empresas

\begin{tabular}{|c|c|c|}
\hline & Empresa A & Empresa B \\
\hline Design como Valor & Sim & Não é percebido como valor \\
\hline Design como estratégia & Sim & Sim, porém não é evidenciado \\
\hline $\begin{array}{l}\text { Fluxograma sistemático } \\
\text { de atividades }\end{array}$ & Sim, domínio sobre o todo & $\begin{array}{l}\text { Sim, porém não tem conhecimento } \\
\text { sobre o todo }\end{array}$ \\
\hline Rotinas & Sim & Sim, através de uso de planilhas \\
\hline Equipe de trabalho & $\begin{array}{l}\text { Gerência, coordenadores } \\
\text { técnicos, assistentes (3) }\end{array}$ & $\begin{array}{l}\text { Diretora (1), gerente (1), } \\
\text { designers (3) }\end{array}$ \\
\hline $\begin{array}{lll}\begin{array}{l}\text { Integração } \\
\text { envolvidos }\end{array} & & \\
\end{array}$ & Sim & $\begin{array}{l}\text { Apenas envolvidos diretos; baixa } \\
\text { integração com a gerência }\end{array}$ \\
\hline Comunicação & $\begin{array}{l}\text { Direta e e-mail; circulava em todos } \\
\text { os níveis; criação de uma } \\
\text { linguagem comum (padronização) }\end{array}$ & $\begin{array}{l}\text { Telefone e email; através de um } \\
\text { supervisor que centralizava as } \\
\text { informações }\end{array}$ \\
\hline Tomadas de decisão & Em equipe & $\begin{array}{l}\text { Equipe desenvolve, mas apenas } \\
\text { uma pessoa decide }\end{array}$ \\
\hline Autonomia & Sim, total & $\begin{array}{l}\text { Para criação sim; } \\
\text { Para tomadas de decisão, não. }\end{array}$ \\
\hline $\begin{array}{l}\text { Cooperação } \\
\text { Colaboração }\end{array}$ & $\begin{array}{l}\text { Sim, desde que não impacte sobre } \\
\text { as entregas }\end{array}$ & Sim, entre a equipe técnica \\
\hline $\begin{array}{l}\text { Competências } \\
\text { individuais }\end{array}$ & $\begin{array}{l}\text { Liderança efetiva, habilidades } \\
\text { interpessoais, líder coaching, } \\
\text { pensamento estratégico e e } \\
\text { orientação para resultados, } \\
\text { conhecimentos técnicos }\end{array}$ & $\begin{array}{l}\text { Boa relação interpessoal, bons } \\
\text { conhecimentos técnicos, agilidade } \\
\text { de pensamento, boa informação } \\
\text { em termos de tendências, } \\
\text { conhecimentos técnicos }\end{array}$ \\
\hline Competências coletivas & $\begin{array}{l}\text { Incentivo ao aprendizado e busca } \\
\text { por uma linguagem comum }\end{array}$ & Trabalho em equipe \\
\hline $\begin{array}{l}\text { Aprendizado (cursos, } \\
\text { palestras, } \\
\text { treinamentos) }\end{array}$ & Sim, promove treinamentos & Incentiva, mas não promove \\
\hline Retenção de recursos & Sim, através de programas internos & Não há preocupação evidente \\
\hline $\begin{array}{l}\text { Banco de dados } \\
\text { (informações sobre o } \\
\text { produto, históricas) }\end{array}$ & Sim, para acesso de todos & Sim, para acesso de todos \\
\hline $\begin{array}{l}\text { Monitoramento de } \\
\text { Satisfação do Cliente }\end{array}$ & Sim & $\begin{array}{l}\text { Somente o gestor, que passava } \\
\begin{array}{l}\text { apenas informações } \\
\text { preferências de cores }\end{array}\end{array}$ \\
\hline
\end{tabular}

Fonte: Elaborado pelos autores, com base na pesquisa realizada. 
Entender o design como estratégia foi um fator evidenciado nas duas entrevistas. Sobre esse tema, nos dois casos apresentados verificou-se a preocupação das empresas e, principalmente o entendimento das entrevistadas em relação ao design como estratégia. No caso da Empresa B, destacou-se que o design como estratégia é essencial, uma vez que representa o potencial de diferenciação a empresa no mercado, assim como uma forma de obter vantagem competitiva diante dos concorrentes.

Já o E2 ocupa o cargo de gerente de estilo da empresa $B$, exercendo atividades ligadas à supervisão de equipe e desenvolvimento de produtos. Na empresa $\mathrm{B}$, embora entende-se o design como estratégia, foi possível verificar que o envolvimento da equipe ocorre, porém, até um determinado nível hierárquico, não havendo autonomia no processo de desenvolvimento dos produtos, tomadas de decisão e solução de problemas. Entretanto, uma característica importante destacada, associada à cultura da empresa foi o investimento em informações em termos de tendências de moda, buscando assim agilidade de pensamento para acompanhamento das tendências da moda mundiais em termos principalmente de criação de estampas para os tecidos.

Conforme os dados obtidos através das entrevistas, foi possível identificar que não há uma padronização do processo de gestão de design entre as empresas. Entretanto, foi possível observar que algumas questões em relação às competências puderam ser identificadas nas duas entrevistas (Quadro 6), vindo de encontro com as questões apresentadas na literatura.

Quadro 6 - Competências identificadas ao longo das entrevistas

\begin{tabular}{|c|c|c|}
\hline \multicolumn{2}{|r|}{ Competências } & Fatores identificados \\
\hline \multirow{3}{*}{ 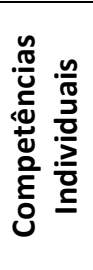 } & Conhecimentos & Técnicos e domínio de conceitos (desenvolvimento de produtos) \\
\hline & Habilidades & $\begin{array}{l}\text { Pensamento estratégico, criatividades, visão voltada ao mercado } \\
\text { (entendimento e aproximação às necessidades dos clientes) }\end{array}$ \\
\hline & Atitudes & $\begin{array}{l}\text { Comprometimento com as tarefas, orientação para resultados, } \\
\text { construção de relacionamentos (integração dos colaboradores), } \\
\text { resolução de problemas (autonomia) }\end{array}$ \\
\hline \multirow{4}{*}{ 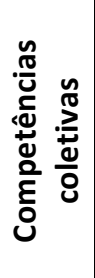 } & Referencial comum & Treinamentos e uso de reuniões com todos os envolvidos \\
\hline & Linguagem compartilhada & $\begin{array}{l}\text { Busca de desenvolvimento de linguagens comuns para facilitar o } \\
\text { entendimento e alinhamento de conceitos }\end{array}$ \\
\hline & Memória Coletiva & Trabalho em equipe, desenvolvendo a discussão e aprendizagem \\
\hline & Engajamento Subjetivo & $\begin{array}{l}\text { Desenvolver a autonomia das pessoas, para que possam realizar as } \\
\text { tomadas de decisão, sem implicar em efeitos indesejados }\end{array}$ \\
\hline
\end{tabular}

Fonte: Elaborado pelos autores, com base na pesquisa realizada.

\subsection{Desenvolvimento da Árvore da Realidade Atual (ARA)}

Para a realização da ARA, foram utilizados os resultados obtidos através do uso do software Mandala (Figura 1) como ponto de partida para a identificação dos principais efeitos indesejados na gestão de design. Dessa forma, foi possível verificar que as palavras mais citadas ao longo das entrevistas foram: design, competências, gestão, valor, cultura e aprendizado.

A ARA (Figura 2) foi desenhada considerando as principais palavras apresentadas na Mandala, assim como com base nas informações obtidas através das entrevistas, buscando identificar quais as causas-raiz para a ausência de uma cultura de gestão de design nas empresas. A partir do desdobramento dos efeitos 
indesejáveis, verificou-se que a principal causa-raiz, com maior impacto sobre os demais fatores está relacionada à falta de competências individuais e coletivas, as quais irão impactar sobre a maior parte dos aspectos necessários para o desenvolvimento de uma cultura em gestão de design.

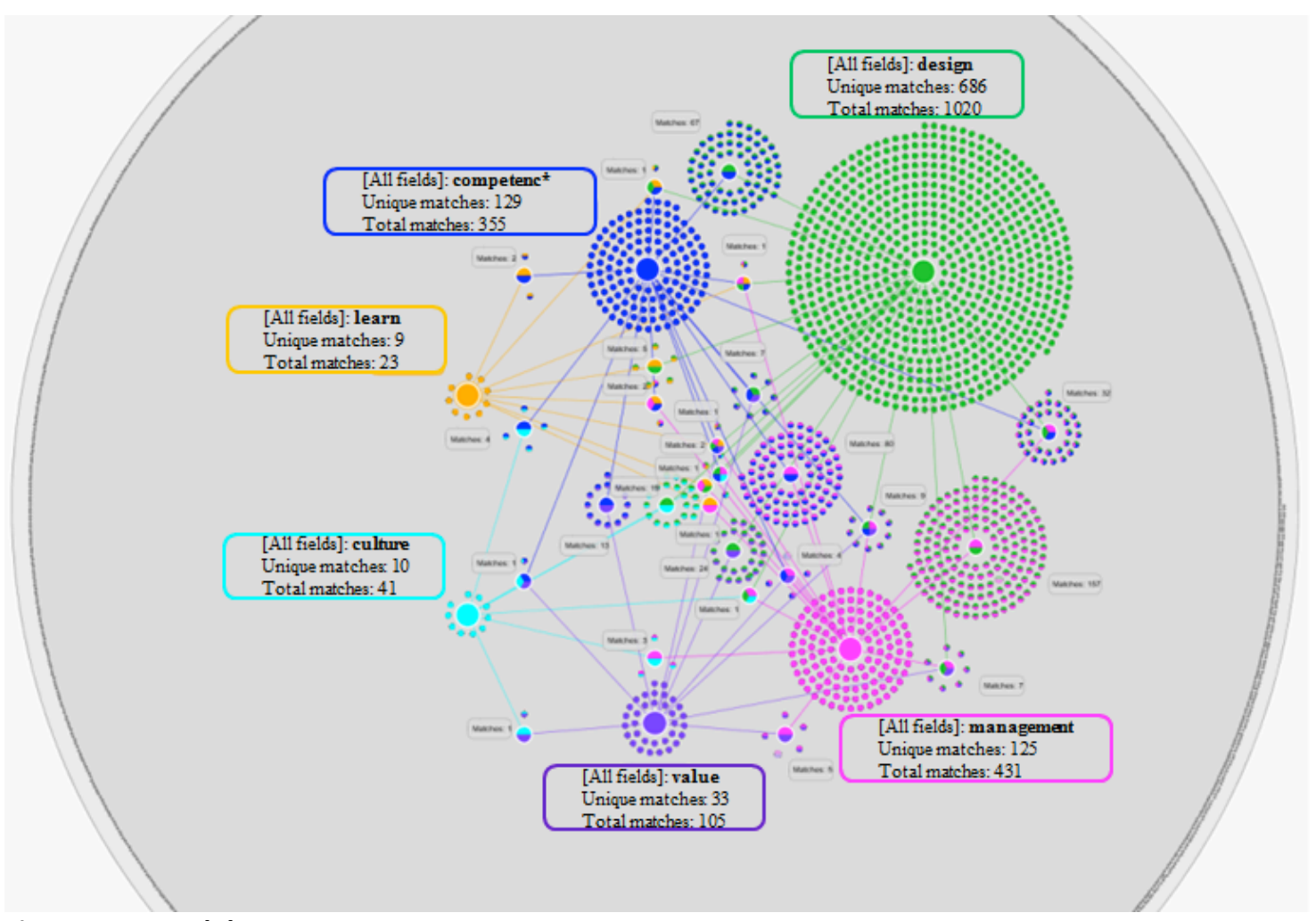

Figura 1 - Mandala

Fonte: Elaborado pelos autores, com base na pesquisa realizada.

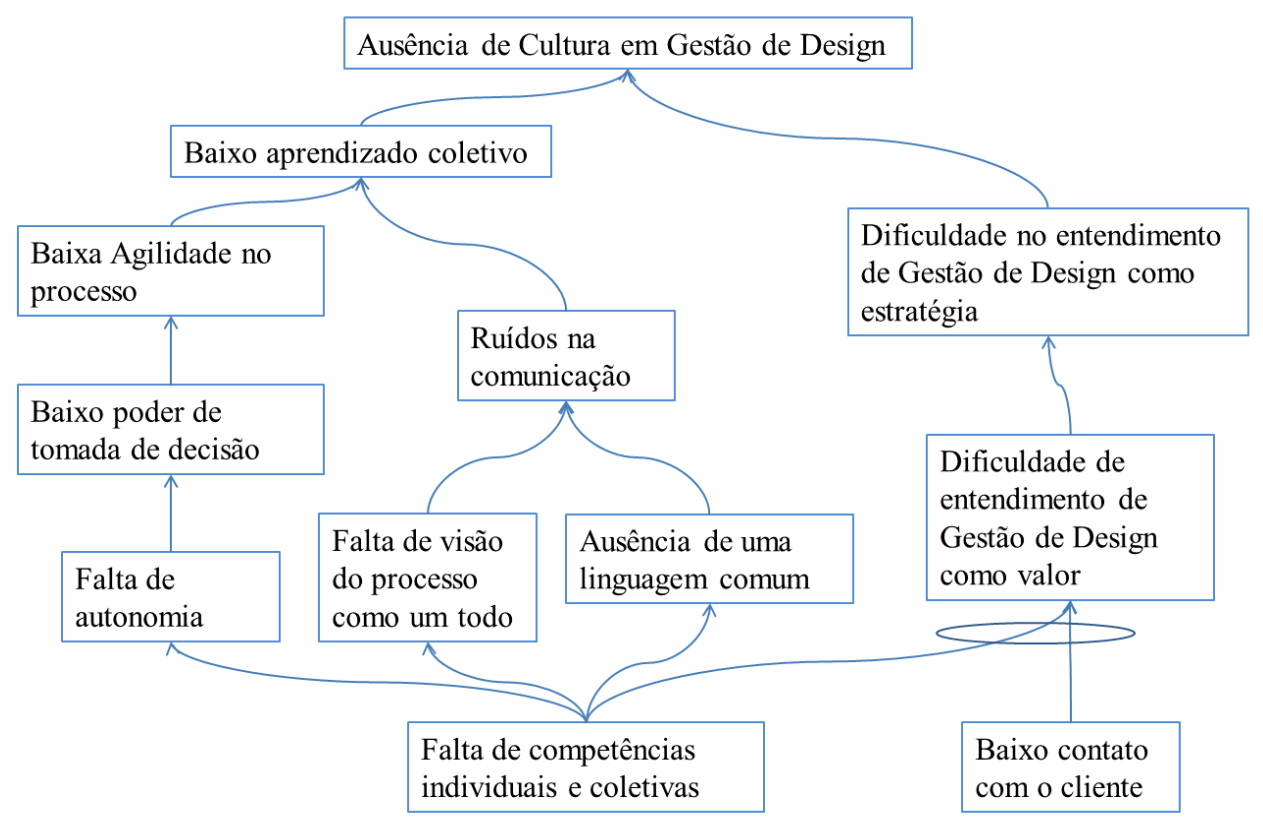

Figura 2 - ARA da gestão de design

Fonte: Elaborado pelos autores, com base na pesquisa realizada. 


\section{CONCLUSÕES}

Este artigo teve como objetivo investigar o que pensam especialistas sobre competências em gestão de design, articulações e fatores intervenientes em duas empresas de grande porte inseridas na indústria do vestuário da região sul do Brasil.

Conforme evidenciado, a indústria do vestuário da região sul do Brasil tem alta representatividade no contexto econômico e social do país, embora apresente vasto campo para desenvolvimento da gestão de design bem como do entendimento do design como um elemento estratégico para a vantagem competitiva sustentável.

A partir dos dados obtidos ao longo da pesquisa, verificou-se que para a obtenção de uma cultura de gestão de design nas empresas, faz-se necessário inicialmente desenvolver as competências individuais e coletivas para que demais efeitos indesejados como falhas de comunicação, baixo aprendizado coletivo e falta de entendimento da gestão de design como valor possam ser eliminados. É importante destacar que a ARA desenhada no presente estudo está relacionada aos casos estudados, não podendo ser generalizada à gestão de design como um todo, embora possa se aplicar.

Desta maneira, na gestão de design de empresas de vestuário, percebe-se claramente a criação e a ocorrência de conhecimento e informação, seja a partir de práticas e rotinas individuais ou mesmo advindas da integração das equipes de trabalho. Arranjos internos, rotinas de trabalho e uma sistemática de processos poderiam auxiliar no mapeamento destas ocorrências para que, assim, seja possível gerenciar as atividades e recursos (tangíveis e intangíveis) envolvidos na gestão de design.

Para pesquisas posteriores, sugere-se a ampliação de estudos para uma amostra maior de empresas, pesquisas em outras regiões do Brasil e, também um estudo para desenvolvimento de uma sistemática de avaliação do conhecimento e da informação para aplicação em empresas da indústria do vestuário.

\section{REFERÊNCIAS}

ABIT - Associação Brasileira de Indústria Têxtil e de Confecções. PRADO, M.V. (org.). Brasil têxtil 2010: relatório setorial da indústria têxtil brasileira. São Paulo: IEMI, 2010.

ABRAVEST - Associação Brasileira da Indústria do Vestuário. Dados Estatísticos do Setor do Vestuário. Disponível na internet por http em <http://www.abravest.org.br>. Acessado em 29 de janeiro de 2014.

BARDIN, L. Análise de conteúdo. ed. rev. e atual. Lisboa: Edições 70, 2005.

BELKADI, F.; BONJOUR, E.; DULMET, M. Competency characterization by means of work situation modeling. Computers in Industry, v. 58, p. 164-178, 2007.

BERTOLA, P.; TEIXEIRA, J.C. Design as a knowledge agent: How design as a knowledge process is embedded into organizations to foster innovation. Design Studies, v. 24, n. 2, p. 181-194, 2003.

BORJA DE MOZOTA, B. Design Management: Using Design to Build Brand Value and Corporate Innovation. New York: Allworth, 2003.

BRUCE, Margaret; COOPER, Rachel; VAZQUEZ, Delia. Effective design management for small businesses. Design Studies, v. 20, 1999. 
DIAS, G.B.; et al.; 2012. In: DUTRA. J.S.; FLEURY, M.T.L.; RUAS, R. Competências: conceitos, métodos e experiências. São Paulo: Atlas, 2012.

GIRARD, P.; ROBIN, V. Analysis of collaboration for project design management. Computers in Industry, v. 57, p. 817-826, 2006.

GOLDRATT, E.M. Theory of Constraints, North River Press, Croton-on-Hudson, NY, 1990.

GUPTA, M.; BOYD, L.; KUZMITS, F. The evaporating cloud: a tool for resolving workplace conflict. International Journal of Conflict Management, v. 22, n. 4, p. 394412, 2011.

KLEINSMANN, M.; VALKENBURG, R. Barriers and enablers for creating shared understanding in co-design projects. Design Studies, v. 29, p. 369-386, 2008.

LACERDA, D.P.; RODRIGUES, L.H.; SILVA, A.C. Uma abordagem de avaliação de processos baseados no mundo dos custos para processos no mundo dos ganhos em instituições de ensino superior. Gestão e Produção, v. 16, n. 4, 2009

LAUCHE, K. Job design for good design practice. Design Studies, v. 26, p. 191-213, 2005.

LEE, K.C.K.; CASSIDY, T. Principles of design leadership for industrial design teams in Taiwan. Design Studies, v. 28, p. 437-462, 2007.

LIBÂNIO, C.S.; AMARAL, F.G. Design Professionals Involved in Design Management: Roles and Interactions in Different Scenarios: A Systematic Review. In: Chakrabarti, A.; Prakash, R.V. (eds.), ICoRD'13, Lecture Notes in Mechanical Engineering, DOI: 10.1007/978-81-322-1050-4_69, Springer India 2013.

MALHOTRA, N. Pesquisa de marketing: uma orientação aplicada. 6.ed. Porto Alegre: Bookman, 2012.

MICHAUX V. Articuler les compétences individuelle, collective, organisationnelle et stratégique: les éclairages de la théorie des ressources et du capital social. In: RETOUR D., PICQ T., DEFELIX C. (eds). Gestion des compétences: Nouvelles relations nouvelles dimensions. Paris: Vuibert - AGRH Gracco CNRS, 13-33, 2009.

MINTZBERG, H.; QUINN, J.B. O Processo da Estratégia. Porto Alegre: Bookman, 2001.

PORTER, M.E. Vantagem Competitiva: criando e sustentando um desempenho superior. Rio de Janeiro: Campus, 1989.

REID, F.J.M. et al. The management of electronics engineering design teams: linking tactics to changing conditions. Design Studies, v. 21, p. 75-97, 2000.

RETOUR, D. Progressos e limites da gestão por competências na França. In: DUTRA, J.S.; FLEURY, M.T.L.; RUAS, R. Competências: conceitos, métodos e experiências. São Paulo: Atlas, 2012.

RETOUR, D.; KROHMER, C. La compétence collective comme maillon clé de la gestion des competences. In: DEFELIX C., KLASFERD A., OIRY E., Nouveaux regards sur la gestion des compétences, Paris, Vuibert, 149-183, 2006.

RUAS, R.L. Competências: compartilhando conceitos e noções. Porto Alegre: Mimeo, 2009. 
RUAS, R.L. Gestão por competências: uma contribuição à estratégia das organizações. In: RUAS, R. L.; ANTONELLO, C. S.; BOFF, L. H. Os novos horizontes da gestão: aprendizagem organizacional e competências. Porto Alegre: Bookman, 2005.

SILVA, F.N.; LIBÂNIO, C.S.; RUAS, R.L. O Estudo das Competências Coletivas em Serviço de Saúde. Negócios e Talentos. v.10, 2013.

YIN, R.K. Estudo de Caso: planejamento e métodos. 2.ed. Porto Alegre: Bookman, 2001. 